\title{
Autoimmune pancreatitis associated to renal and aortic involvement: 3.0-TESLA magnetic resonance imaging in diagnosis and follow-up
}

\author{
Rossella Graziani ${ }^{1}$, Paoletta Preatoni ${ }^{2}$, Silvia Carrara ${ }^{2}$, Alessandro Zerbi ${ }^{3}$, Giovanna Lo Bue ${ }^{1}$, Luca \\ Balzarini $^{1}$
}

1. Department of Radiology, Humanitas Clinical Research Hospital, Rozzano (Milan), Italy. 2 Department of Gastroenterology and Digestive Endoscopy, Humanitas Clinical Research Hospital, Rozzano (Milan), Italy. 3. Department of Surgery, Humanitas Clinical Research Hospital, Rozzano (Milan), Italy

Correspondence: Rossella Graziani. Address: Via D. Frangini, 15-37121-Verona, Italy.

Email: rossella.paola.graziani@gmail.com

Received: February 1, 2015

DOI : $10.5430 /$ crim.v2n3p63
Accepted: July 12, $2015 \quad$ Online Published: July 27, 2015

URL: http://dx.doi.org/10.5430/crim.v2n3p63

\begin{abstract}
Contest: The criteria for Pancreatic Autoimune Pancreatitis (AIP) diagnosis include pancreatic imaging, serological and histopathological findings, other organs involvement and response to steroid treatment.

Case report: Two cases, the first of focal AIP (FAIP), the second of diffuse AIP (DAIP) associated to renal and abdominal aorta involvement respectively, underwent 3.0-Tesla Magnetic Resonance Imaging (3.0-T MRI) with Diffusion Weighted Imaging (DWI) before and after 1 month of corticosteroid treatment. In both cases Computed Tomography, performed for sudden onset of jaundice and pancreatic-like abdominal pain, had previously shown enlargement of pancreatic head and of all pancreatic gland respectively. Endoscopic ultrasound-guided fine-needle aspiration biopsy of both pancreatic lesions had revealed no malignancy. 3.0-T MRI demonstrated a solid mass in enlarged pancreatic head in the first case, an enlargement of all pancreatic gland in the second case, both hypoenhancing in pancreatic phase of contrast enhanced MRI, with moderate delayed enhancement at venous and late phases. Two wedge shaped regions of decreased enhancement of left kidney and a soft-tissue cuffing around the abdominal aorta were observed in first and second case respectively. At DWI, all pancreatic, renal and aortic lesions showed a high signal intensity from restricted diffusion at high $\mathrm{b}$ value $\left(\mathrm{b}=1,000 \mathrm{sec} / \mathrm{mm}^{2}\right)$ images and low apparent diffusion coefficient (ADC) values, compared to the normal ADC value. 3.0-T MRI after treatment, compared to pre-treatment examination, showed marked reduction in size of pancreatic, renal lesions and soft-tissue cuffing aortic lumen. 3.0-T MR DWI revealed an increase of ADC values in all pancreatic, renal and aortic lesions.
\end{abstract}

Conclusion: We emphasize the role of 3.0-T MRI DWI findings and ADC map values on diagnosis of AIP tanks to evaluating the response to short period of steroid treatment of pancreatic and extra pancreatic lesions.

\section{Keywords}

Autoimmune disease, Autoimmune pancreatitis, Magnetic Resonance, Diffusion-weighted Magnetic Resonance, Pancreatitis 


\section{Introduction}

Autoimmune Pancreatitis (AIP) is a form of chronic pancreatitis associated with autoimmune processes. It may be focal or diffuse in all pancreatic gland ${ }^{[1,2]}$. Distinct histological and clinical profile reveal two subtypes of AIP, indistinguishable only on the basis of imaging: the type 1, or lymphoplasmacytic sclerosing pancreatitis (LPSP) and the type 2, or idiopathic duct-centric pancreatitis (IDCP). The type 1 (LPSP) responds characteristically to steroid treatment, though the relapse in the pancreas or other affected organs is common. The most common extra pancreatic site of involvement is the bile duct followed by the retro peritoneum and kidneys ${ }^{[2,3]}$. There are a few reports of IgG4-related arteritis ${ }^{[4-6]}$. In the type 2 (IDCP) the bile duct involvement may be present. Recently, International Consensus Diagnostic Criteria (ICDC) unifies diagnostic criteria of AIP. The comprehensive criteria that must be fulfilled for the diagnosis of disease include pancreatic imaging of parenchyma and ductal system, serological and histopathological findings, other organs involvement and response to steroid treatment ${ }^{[1]}$.

Abdominal Magnetic Resonance imaging (MRI) is a non-invasive, operator independent imaging modality important for the diagnosis of pancreatic disease and AIP ${ }^{[7,8]}$. It is also imaging technique useful for clinicians to visually document alterations of pancreatic gland and other abdominal structures. Images acquired with 1.5-Tesla (1.5-T) MR systems show excellent diagnostic quality. However, with the introduction of clinical 3.0-Tesla (3.0-T) MRI systems in recent years, a shift to the use of higher field strengths has occurred. The improved image quality of 3.0-T MRI compared to 1.5-T MRI depend on the increased signal-to-noise ratio (SNR) and contrast-to-noise ratio (CNR) of higher field strength. Many abdominal applications can benefit from 3.0-T Imaging ${ }^{[9-11]}$, such as MRCP, MR pancreatic study and MR Diffusion-Weighted Imaging (DWI). Delineation of the pancreatic duct, particularly the side branches, is improved at 3.0T MR, compared with 1.5 T MR. 3.0T MR offer improvement in imaging quality also in enhanced study after gadolinium administration because it reduces many of the limitations of $1.5 \mathrm{~T} \mathrm{MR}$, due to artifacts (chemical shift, susceptibility, interference of radiofrequency waves artifacts).The overall quality of DWI obtained at 3.0 T MR is superior to that of DWI obtained at $1.5 \mathrm{~T}$. All these differences may be clinically important.

DWI is a Magnetic Resonance technique measuring changes in the microscopic diffusion of water. The apparent diffusion coefficient (ADC), which can be obtained from MR DWI, is a quantification of the Brownian movement of water molecules. Recently some Authors conclude that pancreatic ADCs obtained with 3.0-T MR DWI may be useful in the diagnosis of Chronic Pancreatitis, Autoimmune Pancreatitis ${ }^{[12]}$ and in differentiation of AIP from Pancreatic Cancer.

In these cases we emphasize the role of 3.0-T MRI, with DWI findings and ADC map values, on diagnosis of focal and diffuse AIP associated to renal and aortic involvement, tanks to evaluating the response to steroid treatment of pancreatic and extra-pancreatic lesions.

\section{Case presentation}

\section{Case 1}

A 72-year-old man, non-smoker and non-drinker, was admitted to our hospital for sudden onset of pancreatic-like abdominal pain associated with jaundice. His past medical history included HCV-related chronic hepatitis, pulmonary tuberculosis when he was 15-year-old, granulomatous disease of mediastinal lymph nodes. He had undergone intestinal surgical resection for small bowel leiomyosarcoma 20 years earlier.

Laboratory investigations on admission showed increased serum levels of bilirubin:16 mg/dl (reference range: 0.2-1.2 $\mathrm{mg} / \mathrm{dl}$ ) with high levels of cholestasis, white blood cells count: $15.0 \times 10^{9} / \mathrm{L}$ (reference range: 4.0-11.0 × 10 $9 / \mathrm{L}$ ), CRP: 67 $\mathrm{mg} / \mathrm{L}$ (reference range: 0-5 mg/L), lipase: $630 \mathrm{UI} / \mathrm{L}$ (reference range: 0-75 UI/L), amylase: $420 \mathrm{UI} / \mathrm{L}$ (reference range: 
40-120 UI/L), IgG4 level: $201 \mathrm{mg} / \mathrm{dl}$ (reference range: 4.8-105 mg/dl, see the Table) and of tumor marker CA19-9: 261 $\mathrm{UI} / \mathrm{ml}$ (reference range: 0-40 UI/ml).

Table. IgG4 serum level before and after steroid treatment in two cases

\begin{tabular}{lll}
\hline IgG4 serum level ( n.v:4.8-105 $\mathbf{~ m g / d )}$ & Before steroid treatment & After steroid treatment \\
\hline Case 1 & 201 & 68 \\
Case 2 & 140 & 45 \\
\hline
\end{tabular}

As shown in the Table, the serum level of IgG4 above the normal value before steroid treatment returned to normal range after treatment in two cases.

A contrast-enhanced Computed Tomography (MDCT) showed a solid hypoenhancing area during pancreatic phase of examination in pancreatic head, less distinguishable from surrounding parenchyma in venous and late phases. Dilation of upstream main pancreatic duct was present. A non-cystic mass lesion hypoenhancing in all phases of examination was visible in parenchyma of left renal upper pole. Endoscopic ultrasound (EUS) confirmed the presence of a hypoechoic area in pancreatic head. Endoscopic ultrasound-guided fine-needle aspiration biopsy (EUS-FNAB) from the lesion of pancreatic head revealed no malignancy, presence of lymphocytic cells and fibrosis.

\section{Case 2}

A 61-year-old man, non-smoker and non-drinker, was admitted to our hospital for sudden onset of pancreatic-like abdominal pain. His past medical and surgical history was unremarkable. Laboratory investigations on admission showed a mild increased serum levels of bilirubin: $2.1 \mathrm{mg} / \mathrm{dl}$ (reference range: 0.2-1.2 mg/dl), a greater increased serum levels of white blood cells count: $16.0 \times 10^{9} / \mathrm{L}$ (reference range: 4.0-11.0 × 10 $/ \mathrm{L}$ ), CRP: $75 \mathrm{mg} / \mathrm{L}$ (reference range: 0-5 mg/L), lipase: $253 \mathrm{UI} / \mathrm{L}$ (reference range: 0-75 UI/L), amylase: $174 \mathrm{UI} / \mathrm{L}$ (reference range: 40-120 UI/L), IgG4 level: $140 \mathrm{mg} / \mathrm{dl}$ (reference range: 4.8-105 mg/dl, see the table). Increased serum level of tumor marker CA19-9 was absent: $30 \mathrm{UI} / \mathrm{ml}$ (reference range: 0-40 UI/ml).

A contrast enhanced MDCT-scan showed enlargement of all pancreatic gland, with a diffuse hypoenhancing parenchyma during pancreatic phase of examination compared to the spleen. Dilatation of main pancreatic duct was absent. Endoscopic ultrasound (EUS) confirmed the presence of diffuse hypoechoic pancreatic parenchyma and fine-needle aspiration biopsy (EUS-FNAB) from the pancreatic gland revealed no malignancy and presence of rare lymphocytic cells.

In Case 1 and Case 2 pancreatic MR and MR Cholangio-Pancreatography (MRCP) imaging were performed on a 3.0-T field strength scanner (Magnetom Verio, Siemens, Erlangen, Germany) using a surphace phased-array body coil. Pancreatic MR imaging included axial T1-weighted fat saturated before and after intravenous contrast medium administration (Gadolinium: GD) with quadriphasic study, axial T2-weighted RARE, axial and coronal T2-weighted Half Fourier sequences, DWI (b value: 0, 50, 600, 1,000 sec/mm²; ADC map), 2D and 3D MRCP.

In Case 1, 3.0-T MR technique performed after MDCT examination, demonstrated a solid mass in enlarged pancreatic head, mildly hyperintense at T2-weighted, hypointense at T1-weighted MR images and hypoenhancing on T1-weighted images after GD administration during arterial pancreatic phase (see Figure 1B), compared to parenchyma of body-tail (see Figure 1A). This lesion showed moderate delayed enhancement at venous and late phases. There were a dilation of upstream main pancreatic duct (see Figure 1A) and smooth stricture in the distal common bile duct. There were also two wedge shaped regions of decreased renal enhancement, at upper (see Figure 1A) and lower (see Figure 1C) pole of left kidney. At axial MR DWI all pancreatic and renal lesions showed a high signal intensity from restricted diffusion at high $b$ value $\left(\mathrm{b}=1,000 \mathrm{sec} / \mathrm{mm}^{2}\right.$ ) images (see Figure 2A-2C ) and low ADC values (see Figure 3A-3C), compared to the ADC value of adjacent normal pancreatic and renal parenchyma (ADC value of $983 \mathrm{sec} / \mathrm{mm}^{2}$ in pancreatic head lesion, compared to ADC value of $1,374 \mathrm{sec} / \mathrm{mm}$ in adjacent normal pancreatic parenchyma of body-tail; ADC value of 895 
$\mathrm{sec} / \mathrm{mm}^{2}$ in upper pole lesion of left kidney and of $845 \mathrm{sec} / \mathrm{mm}^{2}$ in lower pole lesion of left kidney, compared to ADC value of 2,036 sec/ $\mathrm{mm}^{2}$ in adjacent normal parenchyma of left kidney). There was no peripancreatic edema.
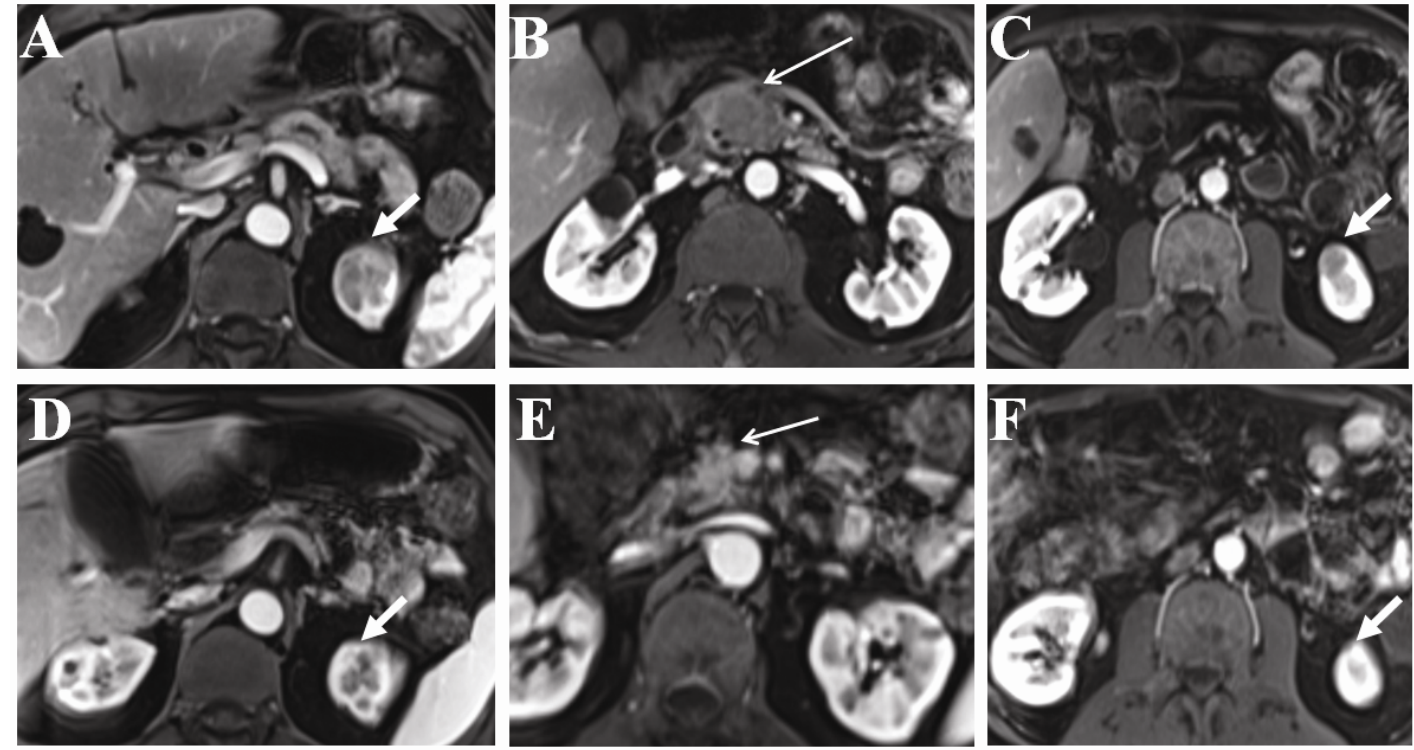

Figure 1. Case 1. 3.0-T MR Imaging before and after steroid treatment. Focal enlargement and two solid, wedge- shaped areas of hypo-enhancement in pancreatic phase of examination were present in pancreatic head (B: arrow), at upper (A: short arrow) and lower (C: short arrow) pole of left kidney. The MR follow-up examination after 1 month high dose steroid treatment showed a reduction in size of pancreatic head with normal parenchyma enhancement during dynamic study (E: arrow), and resolution of abnormal renal cortical enhancement at upper (D: short arrow) and lower pole (F: short arrow) of left kidney
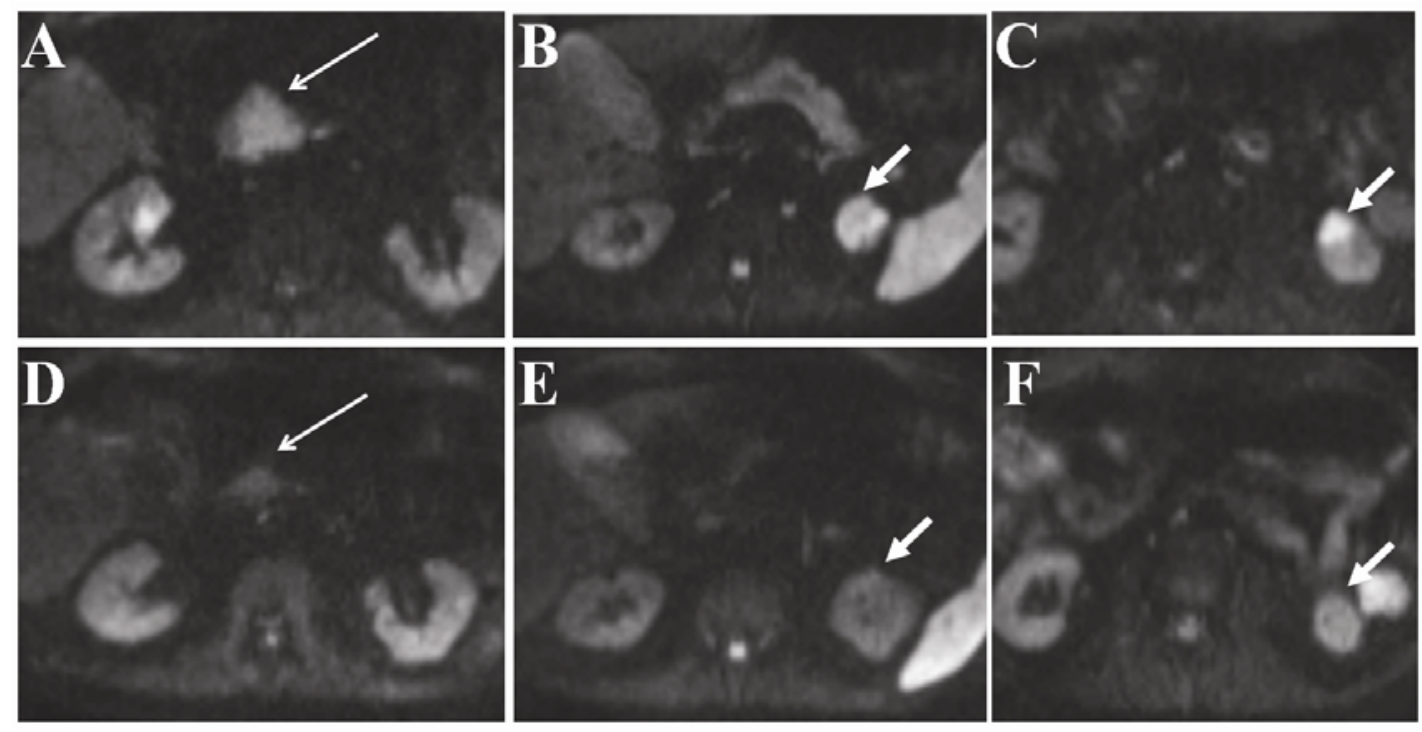

Figure 2. Case1. 3.0-T MR Diffusion weighted imaging before and after steroid treatment. The affected pancreatic parenchyma (A: arrow) and renal parenchyma at upper (B: short arrow) and lower (C: short arrow) pole of left kidney showed restricted diffusion at high $\mathrm{b}$ value $\left(\mathrm{b}=1,000 \mathrm{sec} / \mathrm{mm}^{2}\right.$ ) of 3.0-T MR DW Imaging before steroid treatment, appearing slightly hyperintense in DWI axial images (A-C), due to a severe inflammatory cellular infiltration (lymphocytes, plasmacells). The restricted diffusion at high $b$ value (b = 1,000 $\mathrm{sec} / \mathrm{mm}^{2}$ ) of 3.0-T MR DW Imaging was no visible at the axial DW images in the same pancreatic (D: arrow) and renal areas of upper (E: short arrow) and lower (F: short arrow) pole of left kidney after treatment (D-F) 

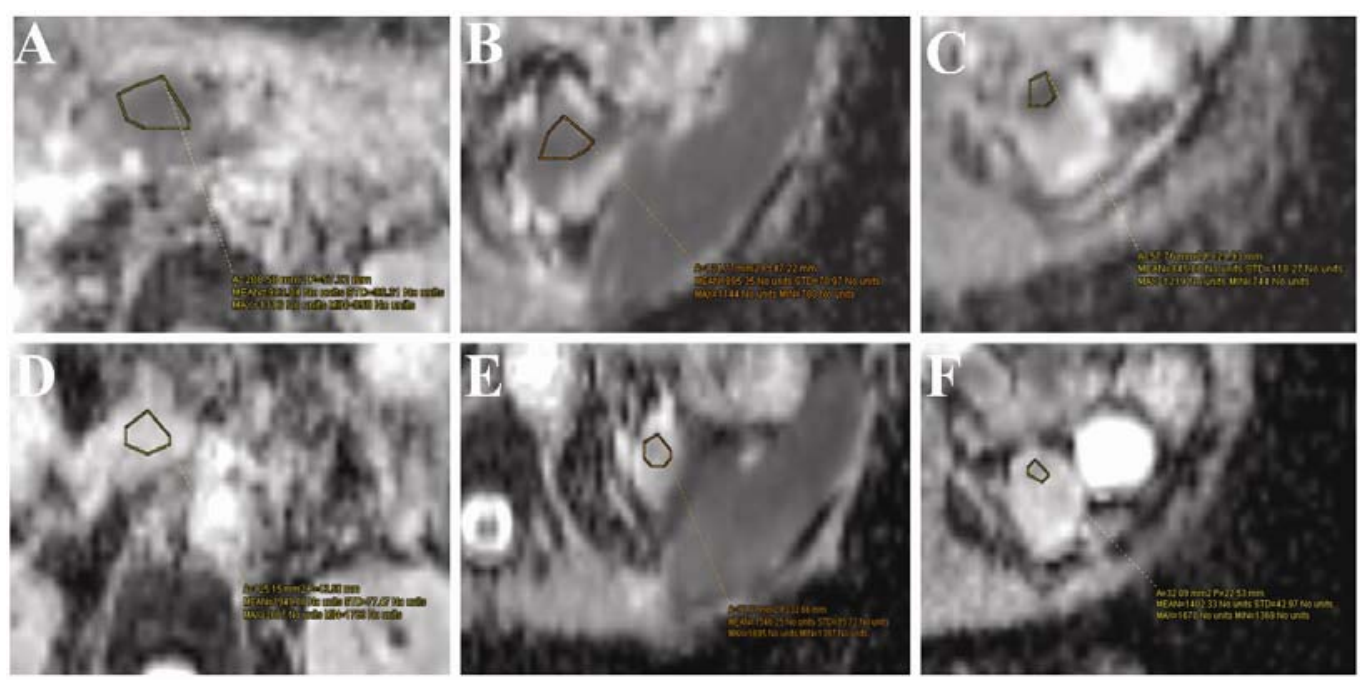

Figure 3. Case 1. 3.0-T MR Diffusion weighted imaging. Axial ADC map before (A-C) and after (D-F) 1 month of steroid treatment. Pre-treatment axial ADC maps values from MR DWI at the level of pancreatic head (A) and upper(B) and lower (C) pole of left kidney were $983 \mathrm{sec} / \mathrm{mm}^{2}, 895 \mathrm{sec} / \mathrm{mm}^{2}, 845 \mathrm{sec} / \mathrm{mm}^{2}$ respectively. Post-treatment ADC values at the same pancreatic(D) and renal areas of upper(E) and lower (F) pole of left kidney increased to $1,949 \mathrm{sec} / \mathrm{mm}^{2}, 1,546 \mathrm{sec} / \mathrm{mm}^{2}, 1,402 \mathrm{sec} / \mathrm{mm}^{2}$ respectively, which is compatible with positive response to treatment

In Case 2, 3.0-T MR technique, performed after MDCT examination, demonstrated a diffuse enlarged of all pancreatic gland, mildly hyperintense at T2-weighted and hypointense at T1-weighted MR images, hypoenhancing on T1weighted images after contrast medium administration during arterial pancreatic phase (see Figure 4A, 4B) compared to parenchyma of spleen. There was also soft -tissue cuffing around the abdominal aorta with decreased enhancement during arterial pancreatic phase (see Figure 4C). At axial MR DWI all pancreatic and aortic lesions showed a high signal intensity from restricted diffusion at high b value $\left(b=1,000 \mathrm{sec} / \mathrm{mm}^{2}\right.$ ) images (see Figure 5A-5C) and low ADC values (see Figure 6A-6C), compared to the ADC value of normal pancreatic gland and normal abdominal aortic wall (ADC value of 1,200 and $939 \mathrm{sec} / \mathrm{mm}^{2}$ in pancreatic body-tail and head respectively; ADC value of 1,127 sec/ $\mathrm{mm}^{2}$ in aortic wall). There was no peripancreatic edema.
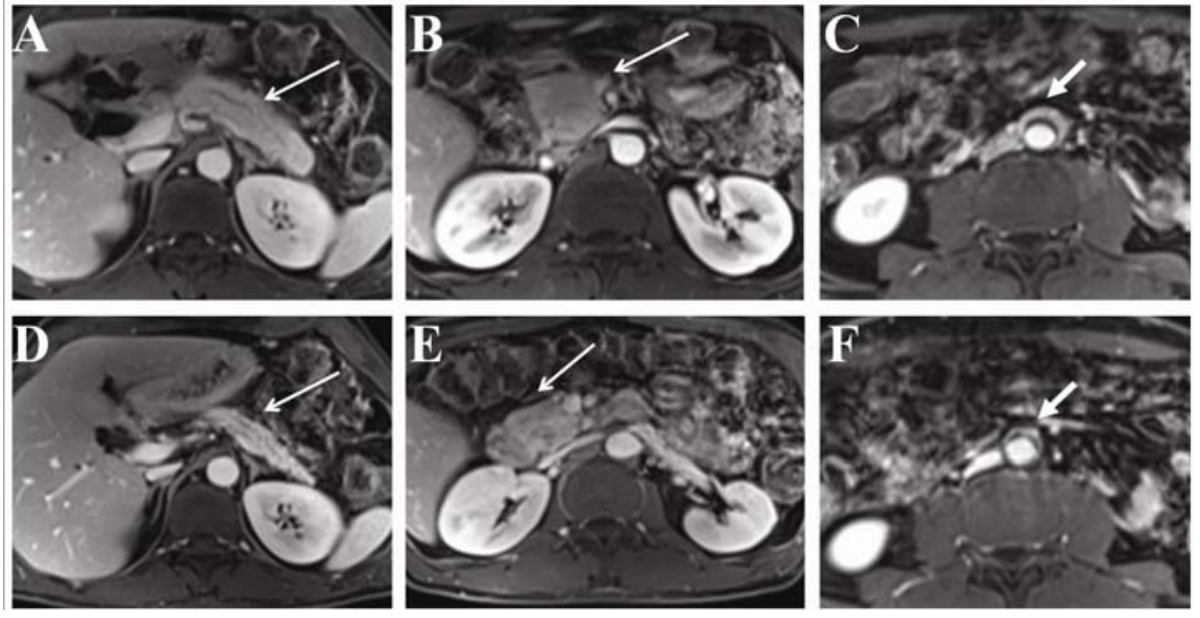

Figure 4. Case 2. 3.0-T MR Imaging before and after corticosteroid treatment. Diffuse enlargement of all pancreatic gland (A, B: arrow) and soft -tissue cuffing around the abdominal aorta (C: arrow), hypo-enhanced in pancreatic phase of examination were present. The MR follow-up examination after 1 month high dose steroid treatment showed a reduction in size of all pancreatic gland with normal parenchyma enhancement during dynamic study (D, E: arrow), and resolution of soft-tissue cuffing around the abdominal abnormal (F: short arrow) 



Figure 5. Case 2. 3.0-T MR Diffusion weighted imaging before and after steroid treatment. The affected pancreatic parenchyma (A-B: arrow) and the affected aortic wall (C: short arrow) showed restricted diffusion at high b value (b=1,000 sec/mm²) of 3.0-T MR DW Imaging before steroid treatment, appearing slightly hyperintense in DWI axial images, due to a severe inflammatory cellular infiltration (lymphocytes, plasmacells). The restricted diffusion at high b value ( $b=1,000 \mathrm{sec} / \mathrm{mm}^{2}$ ) of 3.0-T MR DW Imaging was no visible at the axial DW images in the same pancreatic (D-E: arrow) and aortic wall areas (F: short arrow) after treatment.

The imaging findings were suggestive of Focal Autoimmune Pancreatitis with renal involvement in Case 1 and Diffuse Autoimmune Pancreatitis with aortic involvement in Case 2. Both patients were started on induction corticosteroid treatment with $50 \mathrm{mg}$ of prednisone daily for 4 weeks. One month after corticosteroid treatment the jaundice had regressed in Case 1, pancreatic pain disappeared in Case 1 and 2, serum levels of bilirubin, amylase and lipase were normalized in both patients.

In patient of Case 1 abdominal 3.0-T MR examination and MR DW imaging performed with the same technique of MR imaging before treatment, obtained 1 months after completion of induction corticosteroids, revealed marked reduction in size of solid mass of pancreatic head and more homogeneous enhancement of the parenchyma (see Figure 1E).

There was mild dilation of the upstream main duct, but this had improved compared to his pre-treatment MR finding. Common bile duct remained dilated. The two areas of the abnormal renal cortical enhancement at upper and lower pole of left kidney were not recognizable at MR images (see Figure 1D, 1F) compared to the pre-treatment MR imaging. MR DWI axial images revealed a disappearance of areas of high signal intensity from restricted diffusion (see Figure 2D-2F) at high $\mathrm{b}$ value $\left(\mathrm{b}=1,000 \mathrm{sec} / \mathrm{mm}^{2}\right)$ in pancreatic and renal lesions, of upper and lower pole of left kidney. The ADC values in pancreatic head and left kidney, at upper and lower pole (see Figure 3D-3F) after corticosteroid treatment were increased, compare to the same value of pre-treatment MR technique (from $983 \mathrm{sec} / \mathrm{mm}^{2}$ to $1,949 \mathrm{sec} / \mathrm{mm}^{2}$ in pancreatic head: 98 , $2 \%$ increase; from $895 \mathrm{sec} / \mathrm{mm}^{2}$ to 1,546 in upper pole of left kidney: $72,7 \%$ increase; from $845 \mathrm{sec} / \mathrm{mm}^{2}$ to $1,402 \mathrm{sec} / \mathrm{mm}^{2}$ in lower pole of left kidney: $66 \%$ increase).

For reference the ADC values of the adjacent normal parenchyma after treatment were: $1,578 \mathrm{sec} / \mathrm{mm}^{2}$ in pancreas at body-tail and 1,915 sec/mm in left kidney, comparable to the values before treatment. The serum IgG4 level of patient was $68.0 \mathrm{mg} / \mathrm{dl}$ after steroid treatment which is also still in the normal range (see the Table).

In patient of Case 2 abdominal 3.0-T MR examination and MR DW imaging performed with the same technique of MR imaging before treatment, obtained 1 months after completion of induction corticosteroids, revealed marked reduction in size of all pancreatic gland and more homogeneous enhancement of the parenchyma (see Figure 4D, 4E). 
The soft -tissue cuffing around the abdominal aorta with decreased enhancement was not recognizable at MR images (see Figure 4F) compared to the pre-treatment MR imaging. MR DW axial images revealed a disappearance of areas of high signal intensity from restricted diffusion (see Figure 5D-5F) at high $b$ value $\left(b=1,000 \mathrm{sec} / \mathrm{mm}^{2}\right.$ ) in pancreatic gland and aortic lesions .The ADC values in pancreatic gland (see Figure 6D, 6E) after corticosteroid treatment were increased, compare to the same value of pre-treatment MR technique (from $1,200 \mathrm{sec} / \mathrm{mm}^{2}$ to $1,588 \mathrm{sec} / \mathrm{mm}^{2}$ in pancreatic body-tail: 32, $3 \%$ increase; from $939 \mathrm{sec} / \mathrm{mm}^{2}$ to 1,455 in pancreatic head: $54 \%$ increase). It was not possible to calculate ADC map for each section automatically by the imager software for aortic lesion, because the thin soft-tissue cuffing around the abdominal aorta at MR DWI study before steroid treatment was not well visible at the same MR DWI study after treatment.
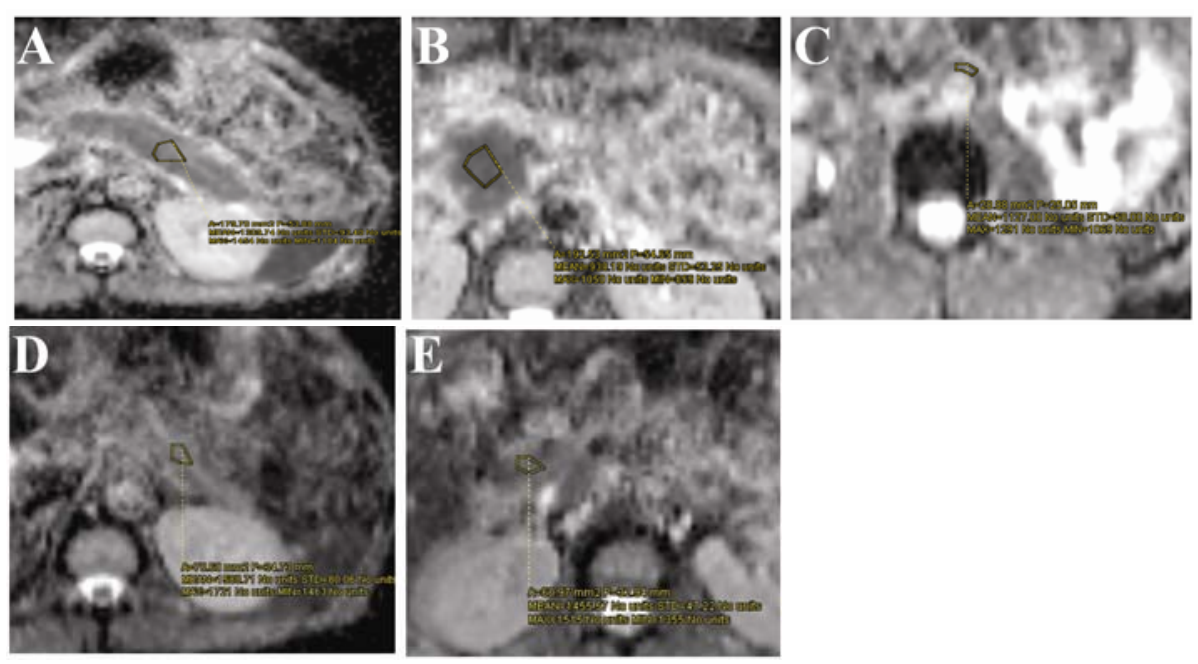

Figure 6. Case 2. 3.0-T MR Diffusion weighted imaging (DWI). Axial ADC map before (A-C) and after (D, E) 1 month of steroid treatment. Pre-treatment axial ADC maps values from MR DWI at the level of pancreatic body-tail (A), pancreatic head (B) and soft-tissue cuffing around the abdominal aorta (C) were 1,200 sec $/ \mathrm{mm}^{2}, 939 \mathrm{sec} / \mathrm{mm}^{2}, 1,127 \mathrm{sec} / \mathrm{mm}^{2}$ respectively. Post-treatment ADC values at the same pancreatic body-tail (D) and pancreatic head (E) increased to 1,588 sec/ $\mathrm{mm}^{2}, 1,455 \mathrm{sec} / \mathrm{mm}^{2}$ respectively, which is compatible with positive response to treatment. Aortic wall involvement was no longer visible in post-treatment ADC map.

Similarly, in Case 2 was not possible to calculate the ADC reference values of the normal parenchyma and aortic wall after treatment and comparable them to the values before treatment. The serum IgG4 level of patient was $45.0 \mathrm{mg} / \mathrm{dl}$ after steroid treatment which is also still in the normal range (see the Table). A diagnosis of autoimmune pancreatitis could be established and confirmed based on the ICDC criteria, which in our patients included cardinal imaging findings, other organ involvement and response to steroid treatment.

\section{Discussion}

Several Authors ${ }^{[7,8]}$ report affected pancreatic parenchyma in AIP shows, as our cases, a decreased enhancement compared to normal pancreatic and splenic parenchyma at enhanced-CT and MR images during pancreatic phase of dynamics study, after intravenous contrast medium administration, like Pancreatic Cancer in Focal AIP, and Pancreatic Lymphoma in Diffuse AIP ${ }^{[12-15]}$.

In Focal AIP, the upstream main pancreatic duct may be dilated, similarly to the case of this report, but it may also be normal. The latter finding is uncommon in patients with Pancreatic Cancer. The MR signal hypointensity at pancreatic phase of dynamic study in affected parenchyma of Focal AIP and Diffuse AIP is reported ${ }^{[7,8,12-15]}$ decreased in the venous and late phases of dynamic study, as our case, and otherwise from Pancreatic Cancer and Pancreatic Lymphoma. Recently some Authors ${ }^{[12]}$ found that the affected pancreatic parenchyma in both diffuse and focal form of AIP appears slightly 
hyperintense in axial MR DWI images with b value $=600 \mathrm{sec} / \mathrm{mm}^{2}$ and baseline ADC value results lower than normal pancreatic parenchyma of patients without chronic pancreatitis. Also in our reports, before treatment, focal lesion of pancreatic head and all pancreatic gland appears slightly hyperintense in axial 3.0-T MR DW images with $b$ value $=1,000$ $\mathrm{sec} / \mathrm{mm}^{2}$ and baseline ADC value results lower than normal pancreatic parenchyma.

Renal lesions are described in approximately 35\% of patients with autoimmune pancreatitis, with the renal parenchyma involved in $30 \%{ }^{[3,8]}$. As in our case, renal parenchyma involvement in AIP patients often result in multiple, bilateral, solid, nodular areas of renal cortex , frequently well-circumscribed and well-shaped, with signal hypointensity at T2 and T1-weighted images and decreased enhancement compared to normal renal cortex in T1-weighted images during dynamics study, after intravenous Gadolinium administration. At histologic analysis of the renal cortex, interstitial nephritis, which progresses to form scars, is present. Nephritis may improve after high dose steroid treatment, with reduction in size of lesions ${ }^{[3,8]}$.

Aortic involvement in AIP patients is rare. There are a few reports of IgG4-related arteritis ${ }^{[4-6]}$. In a study by Kamisawa et al. ${ }^{[16]}$, angiographic abnormalities, including irregular narrowing of the pancreatic arteries, were reported in $54 \%$ of patients with autoimmune pancreatitis. In a study of Vlachou ${ }^{[17]}$, of the 57 AIP patients, 7 (12\%) had arteritis and the aorta was involved in 3 patients, all of them asymptomatic, with soft-tissue cuffing around the affected arteries and mild narrowing and irregularity at imaging. Open biopsy of the abdominal wall is too invasive diagnostic technique. CT and MRI support the diagnosis of chronic aortitis ${ }^{[18,19]}$, that often accompanies peritoneal fibrosis, a lesion recognized in $10 \%$ of patients with AIP. Like AIP, aortitis is caused by immune response to an antigen localized to the aortic adventitia.

Early detection of this disease is important because when aortitis and retroperitoneal fibrosis are advanced, they can lead to aortic rupture. Although we did not have aortic, periaortic and renal tissue histological specimen to study, the efficacy of the steroid treatment suggests that aortic and renal involvement were a manifestation of autoimmune disease in AIP. In all the cases in which AIP is strongly suspected but the diagnosis is uncertain, MR is useful non-invasive, operator independent Imaging modalities to suggest the correct diagnosis because repeated MR examination after short term (2-3 weeks) high dose steroid treatment, can identify a significant reduction in the size of pancreatic parenchyma and the normalization of the caliber of the main pancreatic and bile ducts ${ }^{[3,4,6,7]}$. MR technique can also identify a reduction in size of extra-pancreatic involvement ${ }^{[8,9]}$ more easily than other noninvasive imaging methods such as Ultrasound. The results of this short follow up are obviously present in AIP, like our case, and absent in the case of Pancreatic Cancer and other Pancreatic Neoplasms. The response at short Imaging follow-up after steroid treatment was recently considered diagnostic criteria of AIP ${ }^{[1]}$.

Although MRI sequences, such as T2-weighted and T1-weighted before and after intravenous contrast medium administration, demonstrate anatomic changes in the pancreas and extrapancreatic other organs involvement before and after treatment, Clinicians need to a quantifiable marker for response to therapy.

ADC values have been shown to be a useful imaging biomarker for assessing early treatment response in oncological patients. In our report 3.0-T MR DWI reveals a useful, non-invasive technique for evaluating response to steroid treatment of pancreatic parenchyma and extra pancreatic lesions in AIP, as involvement of kidneys, according to Zaheer ${ }^{[20]}$, and abdominal aortic wall. The ADC, which can be obtained from diffusion weighted MRI, is a quantification of the Brownian movement of water molecules. The presence of inflammation results is restricted diffusion and will be reflected by low ADC values, as in 3.0 T MR DW images of our patients. After steroid treatment, the inflammation is resolved and water molecules are able to freely diffuse across cell membranes resulting in higher ADC values compared to the pre-treatment ADC values ${ }^{[21]}$. In patient of our Cases, the high signal intensity from restricted diffusion at high b value of 3.0-T MR DW Imaging, present before steroid treatment inside pancreatic and extra-pancreatic sites (renal cortex and aortic wall) is no visible at the MR DWI examination in the same pancreatic and extra-pancreatic sites after treatment. The ADC values of the pancreatic and renal lesions markedly increased in our patients after steroid treatment. We assessed 98, 2\%, 72, 7\% , 
66\% increase in pancreatic and renal lesions of Case 1 respectively, compared the ADC value before treatment and 32\%, $54 \%$ increase in pancreatic lesions in Case 2. It was not possible to calculate percentage of increase ADC map values for aortic lesion, because the aortic wall involvement disappeared after steroid treatment.

\section{Conclusion}

3.0-T MRI associated to findings of DWI, having a role in demonstrating and monitoring response to treatment of pancreatic and extra-pancreatic lesions in patients with autoimmune pancreatitis, can be considered a useful, non-invasive, operator independent method helping in the diagnosis of AIP. This imaging modality can allow avoiding radiation exposure of Computed Tomography technique. Pancreatic biopsy is necessary to confirm the diagnosis suggested by MR imaging pattern before treatment. This report suggests the importance of clinical diagnosis using imaging, in particular 3.0-T MR, laboratory and histological tests when AIP is associated with other systemic disorders. Future studies should further evaluate the utility of MRI ADC mapping before and after the administration of steroids in a larger series of patients with pancreatic, renal and aortic lesions in autoimmune pancreatitis.

\section{Conflict of interest}

Authors declare no conflict of interest.

\section{References}

[1] Shimosegawa T, Chari ST, Frulloni L. International Association of Pancreatology. International consensus diagnostic criteria for autoimmune pancreatitis: guidelines of the International Association of Pancreatology. Pancreas. 2011; 40: 352-8. PMid:21412117 http://dx.doi.org/10.1097/MPA.0b013e3182142fd2

[2] Frulloni L, Scattolini C, Falconi M. Autoimmune pancreatitis: differences between the focal and diffuse forms in 87 patients. Am J Gastroenterol. 2009; 104: 2288-2294. PMid:19568232 http://dx.doi.org/10.1038/ajg.2009.327

[3] Yasunari F, Masumi K, Shigeyuki K, et al. Characteristic findings in images of extra-pancreatic lesions associated with autoimmune pancreatitis. European Journal of Radiology. 2010; 76: 228-238. PMid:19581062 http://dx.doi.org/10.1016/j.ejrad.2009.06.010

[4] Matsumoto Y, Kasashima S, Kawashima A, et al. A case of multiple immunoglobulin G4-related periar-teritis: a tumorous lesion of the coronary artery and abdominal aortic aneurysm. Hum Pathol. 2008; 39(6): 975-980. PMid:18430457 http://dx.doi.org/10.1016/j.humpath.2007.10.023

[5] Kamisawa T. Angiographic findings in patients with autoimmune pancreatitis. Radiology. 2005; 236(1): 371; author reply 371-372. PMid:15987989 http://dx.doi.org/10.1148/radiol.2361042057

[6] Kasashima S, Zen Y, Kawashima A, et al. Inflammatory abdominal aortic aneurysm: close relationship to IgG4-related periaortitis. Am J Surg Pathol. 2008; 32(2): 197-204. PMid:18223321 http://dx.doi.org/10.1097/PAS.0b013e3181342f0d

[7] Graziani R, Mautone S, Ambrosetti MC, et al. Autoimmune pancreatitis: multidetector-row computed tomography and magnetic resonance findings in the Italian experience and literature review. Radiol. Med. 2014; 119(8): 558-71. PMid:24638911 http://dx.doi.org/10.1007/s11547-013-0373-9

[8] Manfredi R, Frulloni L, Mantovani W, et al. Autoimmune pancreatitis: pancreatic and extrapancreatic MR imaging-MR cholangiopancreatography findings at diagnosis, after steroid therapy, and at recurrence. Radiology. 2011; 260(2): 428-36. PMid:21613442 http://dx.doi.org/10.1148/radiol.11101729

[9] Chang KJ, Kamel IR, Macura KJ, et al. 3.0-T MR Imaging of the Abdomen: Comparison with 1.5 T. RadioGraphics. 2008; 28: 1983-1998. PMid:19001653 http://dx.doi.org/10.1148/rg.287075154

[10] Patel HT, Shah AJ, Khandelwal SR, et al. MR Cholangiopancreatography at 3.0 T. RadioGraphics. 2009; $29: 1689-1706$. PMid:19959516 http://dx.doi.org/10.1148/rg.296095505

[11] Sandrasegaran K, Lin C, Akisik FM, et al. State-of-the-Art Pancreatic MRI. AJR. 2010; 195: 42-53. PMid:20566796 http://dx.doi.org/10.2214/AJR.10.4421

[12] Taniguchi T, Kobayashi H, Nishikawa K. Diffusion-weighted magnetic resonance imaging in autoimmune pancreatitis. Jpn J Radiol. 2009; 27(3): 138-42. PMid:19412681 http://dx.doi.org/10.1007/s11604-008-0311-2 
[13] Manfredi R, Graziani R, Cicero C, et al. Autoimmune pancreatitis: CT patterns and their changes after steroid treatment. Radiology. 2008; 247: 435-443. PMid:18430876 http://dx.doi.org/10.1148/radiol.2472070598

[14] Graziani R, Frulloni L, Mantovani W, et al. Autoimmune Pancreatitis and non Necrotizing Acute Pancreatitis: Computed Tomography Pattern. Dig. Liver Disease. 2012; 44(9): 759-766. PMID 22546245. PMid:22546245 http://dx.doi.org/10.1016/j.dld.2012.03.013

[15] Takahashi N, Kawashima A, Fletcher JG. Renal involvement in patients with autoimmune pancreatitis: CT and MR imaging findings. Radiology. 2007; 242: 791-801. PMid:17229877 http://dx.doi.org/10.1148/radiol.2423060003

[16] Kamisawa T, Egawa N, Nakajima H, et al. Clinical difficulties in the differentiation of autoimmune pancreatitis and pan-creatic carcinoma. Am J Gastroenterol. 2003; 98(12): 2694-2699. PMid:14687819 http://dx.doi.org/10.1111/j.1572-0241.2003.08775.x

[17] Vlachou PA, Khalili K, Jang H-J, et al. IgG4-related sclerosing disease: Autoimmune Pancreatitis and extrapancreatic manifestations. Radio Graphics. 2011; 31: 1379-1402. http://dx.doi.org/10.1148/rg.315105735

[18] Jois RN, Gaffney K, Marshall T, et al. Chronic periaortitis. Rheumatology (Oxford). 2004; 43: 1441-1446. PMid:15266064 http://dx.doi.org/10.1093/rheumatology/keh326

[19] Matsubayashia H, Furukawab, Uesakac K, et al. Autoimmune Pancreatitis Accompanied by Cholecystitis, Periaortitis and Pseudotumors of the Liver. Case Rep Gastroenterol. 2008; 2: 155-161. http://dx.doi.org/10.1159/000125456

[20] Zaheer A, Halappa VG, Akshintala VS. Renal lesions in autoimmune pancreatitis: diffusion weighted magnetic resonance imaging for assessing response to corticosteroid therapy. JOP. 2013; 14(5): 506-509. PMid:24018596

[21] Yukutake M, Sasaki T, Serikawa M, et al. Timing of radiological improvement after steroid therapy in patients with autoimmune pancreatitis. Scand J Gastroenterol. 2014; 49(6): 727-33. PMid:24665967 http://dx.doi.org/10.3109/00365521.2014.900822 